\title{
ON FINITE PROJECTIVE GAMES
}

\author{
MOSES RICHARDSON ${ }^{1}$
}

1. Preliminaries on simple games. Let $N=\{1,2, \cdots, n\}$ be a finite set of $n$ elements termed players. Let $\Re$ be the class of all subsets $S$ of $N$; the elements $S$ of $\Re$ are termed coalitions. If $S \subset \Re$, let $S^{+}$denote the class of all supersets of elements of $\delta$, and $\delta^{*}$ the class of all complements of elements of $s$; in symbols, $s^{+}=[X \in \Re \mid X \supset S$ for some $S \in \mathcal{S}], \mathcal{S}^{*}=[X \in \mathfrak{X} \mid N-X \in \mathcal{S}]$. By a simple game is meant an ordered pair $G=(N, W)$ where $W \subset \mathfrak{T}$ satisfies (1) $W=W^{+}$, (2) $W^{*} W^{*}=0$. The elements of $W$ are termed winning coalitions. The elements of $\mathscr{L}=\mathfrak{T}-\mathscr{W}$ are termed losing coalitions. The elements of $B=\mathscr{L} \cap \mathfrak{L}^{*}$ are termed blocking coalitions. A simple game ${ }^{2}$ is termed strong if $B=0$. A simple game may be defined by specifying the class $W^{m} C W$ of minimal winning coalitions. By an imputation is meant an ordered $n$-tuple of real numbers $x=\left(x_{1}, x_{2}, \cdots, x_{n}\right)$ such that ${ }^{3}$ $x_{i} \geqq 0$ and $\sum_{i=1}^{n} x_{i}=1$. If $u \subset \Re$, let $\mathfrak{u}^{0}=\mathfrak{U}-\left(\mathfrak{u}^{+}\right)^{*} ; \mathfrak{u}^{0}$ is the class of all coalitions which intersect every element of $\mathcal{u}$. If $\mathfrak{U}=\mathfrak{w}^{m}$ then $\mathcal{u}^{0}=\mathscr{L}^{*}=W_{\mathcal{U}}$.

Suppose given a simple game $(N, w)$, a nonempty class $u \subset w$, and real numbers $a_{1}, a_{2}, \cdots, a_{n}$ such that

$$
\begin{aligned}
& \sum_{i \in S} a_{i}=1 \text { for } S \in \mathcal{u}, \\
& \sum_{i \in S} a_{i}>1 \text { for } S \in \mathcal{u}^{0}-\mathcal{u} .
\end{aligned}
$$

Let $x^{(S)}$ denote the imputation of which the $i$ th component is $a_{i}$ if $i \in S$ and 0 otherwise. Then the finite set of imputations $X=\left[x^{(s)} \mid S\right.$ $\in \mathfrak{U}]$ is termed a simple solution of the game $(N, w)$. If $\mathfrak{u}=W^{m}$ then $X$ is termed a main simple solution (cf. [4], pp. 443-444).

2. Finite projective games. The following remarks stem from

Presented to the Society, April 16, 1955; received by the editors November 4, 1954 and, in revised form, June 19, 1955.

${ }^{1}$ This work was done for the Logistics Project sponsored by the Office of Naval Research in the Department of Mathematics, Princeton University.

2 The original definition of simple games in von Neumann and Morgenstern [4] is such as to forbid the existence of blocking coalitions. Thus the simple games of [4] are our strong simple games. The definitions used here are due to Shapley [5].

${ }^{3}$ We use the $(0,1)$-normalization. The precise relationship, not needed for reading this paper, between this normalization and that of [4] can be found explicitly in [2] or [3]. 
curiosity concerning a footnote in von Neumann and Morgenstern $[4$, p. 469 , footnote 3$]$, to the effect that finite projective geometries other than the seven-point one seem unsuitable for the "present purpose" of providing examples of simple games. The explanation of this statement is given by Theorem 1 below, in view of our footnote 2 .

Consider the $k$-dimensional projective space $P G\left(k, p^{n}\right)$ whose field of coordinates is the Galois field $G F\left(p^{n}\right)$ where $p$ is prime and $n$ a positive integer. ${ }^{4}$ We define a simple game based on this space as follows. The players shall be all the points of the $k$-space. Since no two winning coalitions can be complementary, it is essential to define the game by choosing the minimal winning coalitions so that any pair of them intersect. Since an $l$-space and an $m$-space in a projective $k$-space must intersect if $l+m \geqq k$, it is natural to select as minimal winning coalitions the linear subspaces of lowest dimension such that they all intersect pairwise. Thus, if $k$ is even, $k=2 h$, let the $h$-spaces be chosen; and if $k$ is odd, $k=2 h+1$, let the $(h+1)$ spaces be chosen. The simple game thus defined will be denoted also by $P G\left(k, p^{n}\right)$ and will be termed a finite projective game.

A blocking coalition is one which is not winning but which intersects every winning coalition. Clearly, if $k=2 h+1$, all the $h$-spaces are blocking coalitions. These blocking coalitions have fewer members than the minimal winning coalitions, since the number of points in a $q$-space is $1+p^{n}+p^{2 n}+\cdots+p^{q n}$ (cf. the corollary to Theorem 2 below). The remainder of this note confines itself to the simplest even-dimensional case, namely the finite projective plane games $P G\left(2, p^{n}\right)$. Here, the lines are the minimal winning coalitions, and a blocking coalition is a set of points containing no line but intersecting every line.

Theorem 1. The game $P G\left(2, p^{n}\right)$ is strong if $p^{n}=2$, and not strong if $p^{n}>2$. In particular, there exists a blocking coalition of $2 p^{n}$ players if $p^{n}>2$.

Proof. Choose an arbitrary point $b_{1}$ as the first member of the proposed blocking coalition $B$. It intersects $1+p^{n}$ lines of the plane. Let $l$ be one of these lines and let $b_{2}, b_{3}, \cdots, b_{p^{n}}$ be distinct points of $l$ different from $b_{1}$. Each of the points $b_{i}\left(i=2,3, \cdots, p^{n}\right)$ intersects $p^{n}$ lines different from $l$. Together the set of points $\left(b_{1}, b_{2}, \cdots, b_{p^{n}}\right)$ intersect $\left(1+p^{n}\right)+\left(p^{n}-1\right) p^{n}=p^{2 n}+1$ lines. Let $a$ be the $\left(p^{n}+1\right)$ th point of $l ; a$ cannot be put into $B$. There are $p^{n}$ lines left unintersected by the points so far put into $B$, all these lines containing $a$.

${ }^{4}$ Notation and basic facts concerning these finite projective spaces are due to Veblen and Bussey [8]. Another exposition can be found in Carmichael [1]. 
There are $p^{2 n}$ points of the plane not on $l$ not yet used, $p^{n}$ of them on each of the $p^{n}$ lines through $a$ just mentioned. We shall show that $p^{n}>2$ is a necessary and sufficient condition that we can choose one point on each of these $p^{n}$ lines to put into $B$ so that no $p^{n}+1$ points of $B$ are collinear. There are $p^{n} \cdot p^{n} \cdots p^{n}=\left(p^{n}\right)^{p^{n}}=p^{n p^{n}}$ available $p^{n}$-tuples of points that can be chosen so as to intersect the remaining $p^{n}$ lines. If $p^{n}>2$, then $p^{n p^{n}}>p^{2 n}$. The points $b_{1}, b_{2}, \cdots, b_{p^{n}}$ have intersected only $p^{2 n}$ lines other than $l$. Therefore not all these $p^{n p^{n}} p^{n}$ tuples can colline with any of the points $b_{1}, \cdots, b_{p^{n}}$. Hence there exists a $p^{n}$-tuple which together with the points $b_{1}, \cdots, b_{p^{n}}$ constitute a blocking coalition $B$ of $2 p^{n}$ members, if $p^{n}>2$. If $p^{n}=2$, this is impossible, cf. Figure 1. For the two remaining lines meeting at $a$ contain 4 other points, say $x$ and $y$ on one line, and $x^{\prime}$ and $y^{\prime}$ on the other.

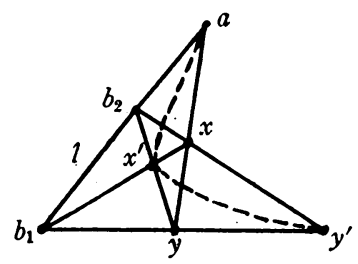

FIG. 1

Each of the 4 possible pairs $x x^{\prime}, x y^{\prime}, x^{\prime} y$, or $y y^{\prime}$ collines with a used point of $l$. Therefore there exists no 4-person blocking coalition in this 7 -point geometry $P G(2,2)$, and in fact no blocking coalition at all. This completes the proof.

\section{Simple solutions.}

TheOREM 2. If $B$ is a blocking coalition in $P G\left(2, p^{n}\right), p^{n}>2$, then the number $|B|$ of members of $B$ is greater than the number $1+p^{n}$ of points on a line.

Proof. Case 1. If $p^{n}$ of the points of $B$ are on some line $l$ then they intersect $\left(p^{n}+1\right)+\left(p^{n}-1\right) p^{n}=p^{2 n}+1$ lines, leaving $p^{n}$ lines unintersected so far. A $\left(p^{n}+1\right)$ th point not on $l$ can then intersect only one new line. Since $1<p^{n}$, not all lines are intersected by these $p^{n}+1$ points and $|B| \geqq p^{n}+2$.

Case 2. Suppose the maximum number of collinear points in $B$ is less than $p^{n}$. Then any $p^{n}$ points of $B$ intersect fewer than $p^{2 n}+1$ lines since at least one of them must intersect fewer than $p^{n}$ new lines. Then more than $p^{n}$, i.e. at least $p^{n}+1$, lines are left unintersected. But the $\left(p^{n}+1\right)$ th point of $B$ cannot intersect more than $p^{n}$ new lines. 
Hence at least one line is still left unintersected and hence $|B|>p^{n}$ +1 . This completes the proof.

Corollary. Every two-dimensional finite projective game $P G\left(2, p^{n}\right)$ has a main simple solution. ${ }^{5}$

Proof. Let $a_{i}=1 /\left(p^{n}+1\right)$. There exists a simple solution consisting of one imputation for each line or minimal winning coalition $S$ assigning $a_{i}$ to $i \in S$ and 0 to $i \in-S$. Our Theorem 2, above, implies condition (ii) of the definition of simple solution, namely $\sum_{i \in S} a_{i}>1$ for $S \in\left(W_{\mathcal{W}} \cup()_{)}-W^{m}\right.$, if $p^{n}>2$. The case $p^{n}=2$ is disposed of in [4, p. 469]. This completes the proof. ${ }^{6}$

4. Blocking coalitions. If $\min |B|$ is the minimum number of members in a blocking coalition in $P G\left(2, p^{n}\right), p^{n}>2$, then we have established that $p^{n}+2 \leqq \min |B| \leqq 2 p^{n}$. It would be of interest to sharpen this result for $P G\left(2, p^{n}\right)$ by determining what min $|B|$ is exactly. The following fragmentary results bear on this problem.

THEOREM 3. If a set $S$ of points of $P G\left(2, p^{n}\right)$ contains fewer than $2 p^{n}$ members and if $p^{n}$ of the points of $S$ are collinear but $S$ contains no line, then the complementary set $-S$ contains at least one entire line.

Proof. Let the points $s_{1}, s_{2}, \cdots, s_{p^{n}}$ of $S$ all lie on a line $l$. Then they intersect $p^{2 n}+1$ lines. Any further point of $S \cap(-l)$ intersects just one line not intersected by $S \cap l$, namely the line determined by that point and $l \cap(-S)$, and hence intersects at most one new line. Hence if $|S| \leqq 2 p^{n}-1$, the number of intersected lines is not greater than $\left(p^{2 n}+1\right)+\left(p^{n}-1\right) \cdot 1=p^{2 n}+p^{n}$. This leaves at least one line not intersected by $S$, hence contained in $-S$.

COROLlARY. The minimum number of elements in a blocking coalition of $P G\left(2, p^{n}\right), p^{n}>2$, which has $p^{n}$ collinear points in it, is $2 p^{n}$.

However $2 p^{n}$ is not in general the minimum number of elements in a blocking coalition of $P G\left(2, p^{n}\right)$. We show below that it is so for $P G(2,3)$, but not for $P G(2,4)$; in the latter case we exhibit a 7 -point blocking coalition.

THEOREM 4. In $P G(2,3)$, the minimum number of elements in a blocking coalition is 6 .

${ }^{5}$ The author is indebted to L. S. Shapley for pointing out this corollary in conversation.

${ }^{6}$ We note parenthetically that $\sum_{i \in N} a_{i}=1+p^{2 n} /\left(1+p^{n}\right)>2$; compare $(50: 21)$ of p. 445 of [4] where the $2 n$ is now replaced by 2 because of our use of the $(0,1)$ normalization. See also [5]. Also parenthetically, it follows from Theorem 4 of [3] that $P G\left(2, p^{n}\right)$ is $k$-unstable for $p^{n} \leqq k<p^{n}+p^{2 n}$ and $k$-stable for $1 \leqq k<p^{n}$. 
Proof. We use the cyclic representation ${ }^{7}$ of $P G(2,3)$ :

$\begin{array}{rrrrrrrrrrrrr}0 & 1 & 2 & 3 & 4 & 5 & 6 & 7 & 8 & 9 & 10 & 11 & 12 \\ 1 & 2 & 3 & 4 & 5 & 6 & 7 & 8 & 9 & 10 & 11 & 12 & 0 \\ 3 & 4 & 5 & 6 & 7 & 8 & 9 & 10 & 11 & 12 & 0 & 1 & 2 \\ 9 & 10 & 11 & 12 & 0 & 1 & 2 & 3 & 4 & 5 & 6 & 7 & 8\end{array}$

in which the points are denoted by $0,1, \cdots, 12$ and the lines consist of the points in the vertical columns.

Put an arbitrary point $b_{1}$ into the proposed blocking coalition $B$; it intersects 4 lines. Put any point $b_{2} \neq b_{1}$ into $B$; it intersects 3 new lines. Put $b_{3} \neq b_{1}, b_{2}$ into $B ; b_{3}$ may be (A) on the line $b_{1} b_{2}$ or (B) not. In case $(\mathrm{A}), b_{3}$ intersects 3 new lines with a cumulative total of 10 lines intersected. In case (B), $b_{3}$ intersects 2 new lines for a total of 9 lines intersected. Put $b_{4} \neq b_{1}, b_{2}, b_{3}$ into $B$. In case (A), $b_{4}$ may not be collinear with $b_{1}, b_{2}, b_{3}$ because, if so, $\left(b_{1}, b_{2}, b_{3}, b_{4}\right)$ is a line and hence a minimal winning coalition, not a blocking coalition; hence $b_{4}$ is not thus collinear with $b_{1}, b_{2}, b_{3}$ and therefore intersects one new line, for a total of 11 lines intersected. In case (B), $b_{4}$ may be: case (B1) on one of the lines $b_{1} b_{2}, b_{1} b_{3}$, or $b_{2} b_{3}$ in which case $b_{4}$ intersects 2 new lines for a total of 11 ; or case (B2) if $b_{4}$ is not on any of these 3 lines, then $b_{4}$ intersects one new line for a total of 10 lines intersected. Hence there exists no 4 -person blocking coalition. Put $b_{5} \neq b_{1}, b_{2}, b_{3}, b_{4}$ into $B$. In case (A), $b_{5}$ may not colline with $b_{1}, b_{2}, b_{3}$, as before; hence $b_{5}$ intersects at most one new line for a total of either 11 or 12 . In case (B1), $b_{5}$ may not colline with $b_{1}, b_{2}, b_{4}$, say, for then $\left(b_{5}, b_{4}, b_{2}, b_{1}\right)$ would be a line and hence not blocking, and hence yields a total of 12 at most. In case (B2), either: (i) $b_{5}$ is on one of the lines $b_{1} b_{2}$, $b_{1} b_{3}, b_{1} b_{4}, b_{2} b_{3}, b_{2} b_{4}, b_{3} b_{4}$ or on two of them, so that $b_{5}$ intersects 1 or 2 new lines, respectively, for a total of 11 or 12 lines intersected; or (ii) if $b_{5}$ is on none of these lines then it intersects no new line, for a total of 10 lines intersected. Hence there exists no 5-person blocking coalition. The set $(0,1,5,6,7,11)$ is a 6 -person blocking coalition. This completes the proof.

In $P G(2,4)$, we shall exhibit a 7 -person blocking coalition. The cyclic representation of $P G(2,4)$ is:

$\begin{array}{rrrrrrrrrrrrrrrrrrrrr}0 & 1 & 2 & 3 & 4 & 5 & 6 & 7 & 8 & 9 & 10 & 11 & 12 & 13 & 14 & 15 & 16 & 17 & 18 & 19 & 20 \\ 1 & 2 & 3 & 4 & 5 & 6 & 7 & 8 & 9 & 10 & 11 & 12 & 13 & 14 & 15 & 16 & 17 & 18 & 19 & 20 & 0 \\ 4 & 5 & 6 & 7 & 8 & 9 & 10 & 11 & 12 & 13 & 14 & 15 & 16 & 17 & 18 & 19 & 20 & 0 & 1 & 2 & 3 \\ 14 & 15 & 16 & 17 & 18 & 19 & 20 & 0 & 1 & 2 & 3 & 4 & 5 & 6 & 7 & 8 & 9 & 10 & 11 & 12 & 13 \\ 16 & 17 & 18 & 19 & 20 & 0 & 1 & 2 & 3 & 4 & 5 & 6 & 7 & 8 & 9 & 10 & 11 & 12 & 13 & 14 & 15 .\end{array}$

7 The existence of such a cyclic representation for all $P G\left(k, p^{n}\right)$ was established by J. Singer [6]. Another proof appears in E. Snapper [7]. 


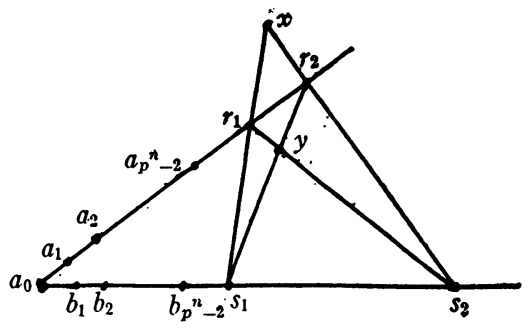

FIG. 2

Then the set $(0,1,4,5,6,15,20)$ is a 7 -person blocking coalition. This illustrates the following theorem.

THEOREM 5. If $p^{n}>3$, there exists in $P G\left(2, p^{n}\right)$ a blocking coalition of $2 p^{n}-1$ members.

Proof. Cf. Figure 2. Let $a_{0}, a_{1}, \cdots, a_{p_{n-2}}$ be distinct collinear points, and let $b_{1}, b_{2}, \cdots, b_{p^{n-2}}$ be distinct points collinear with $a_{0}$ but not on the line $a_{0} a_{1}$. These $2 p^{n}-3$ points intersect $\left(p^{n}+1\right)$ $+\left(p^{n}-2\right) p^{n}+\left(p^{n}-2\right) 2=p^{2 n}+p^{n}-3$ lines. Let $r_{1}, r_{2}$ be the remaining points on $a_{0} a_{1}$ and $s_{1}, s_{2}$ the remaining points on $a_{0} b_{1}$. Then let $x=r_{1} s_{1} \cap r_{2} s_{2}$ and $y=r_{1} s_{2} \cap r_{2} s_{1}$. The points $x, y$ intersect the four remaining lines $r_{1} s_{1}, r_{2} s_{1}, r_{1} s_{2}, r_{2} s_{2}$. Hence the set $B=\left[a_{0}, a_{1}, \cdots, a_{p^{n-2}}\right.$, $\left.b_{1}, b_{2}, \cdots, b_{p^{n-2}}, x, y\right]$ will constitute a blocking coalition of $2 p^{n}-1$ points unless it contains a line. Now the collinear points $a_{0}, a_{1}, \cdots$, $a_{p^{n-2}}$ fall short of a line by two points, as do the collinear points $a_{0}, b_{1}, \cdots, b_{p^{n-2}}$. The points $x$ and $y$ are on neither of the two lines $a_{0} a_{1}$ and $a_{0} b_{1}$. Finally $x$ and $y$ colline with at most two points $a_{i}, b_{j}$ of $B$, one from each of these two lines. (Note that $x$ and $y$ may colline with only one point $a_{0}$ of these two lines, since the diagonal points of a complete quadrangle colline if and only if $p=2$, but this does not affect our argument; cf. [8] or [1].) Hence the set $B$ is a blocking coalition unless the set $\left(x, y, a_{i}, b_{j}\right)$ contains a line, which can happen only if $p^{n}+1 \leqq 4$, or $p^{n} \leqq 3$. This completes the proof.

That Theorem 5 does not provide the minimum number of elements in a blocking coalition is shown by the next theorem.

THEOREM 6. If $d$ is a divisor of $n, 1 \leqq d<n$, then there exists in $P G\left(2, p^{n}\right)$ a blocking coalition $B$ with $2 p^{n}-p^{d}+1$ members.

Proof. In $P G\left(2, p^{d}\right)$, let $a_{0}, r_{1}, r_{2}, \cdots, r_{p^{d}}$ be the points of one line, let $a_{0}, s_{1}, s_{2}, \cdots, s_{p^{d}}$ be the points of a second line, and let $a_{0}, x_{1}$, $x_{2}, \cdots, x_{p d}$ be the points of a third line through $a_{0}$. The set $X=\left[x_{1}, x_{2}, \cdots, x_{p^{d}}\right]$ clearly intersects all of the $p^{2 d}$ lines $r_{i} s_{j}$. 
Since $d$ is a divisor of $n$ and $1 \leqq d<n, P G\left(2, p^{d}\right)$ can be imbedded (cf. [8] or [1]) in $P G\left(2, p^{n}\right)$. Let $L_{r}\left(L_{s}\right)$ be the line of $P G\left(2, p^{n}\right)$ containing the points $r_{i}\left(s_{j}\right)$. Let $A=\left[a_{1}, a_{2}, \cdots, a_{p^{n}-p^{d}}\right]$ be the set of points of $L_{r}$ not in $P G\left(2, p^{d}\right)$, and let $C=\left[c_{1}, c_{2}, \cdots, c_{p^{n}-p^{d}}\right]$ be the set of points of $L_{s}$ not in $P G\left(2, p^{d}\right)$. Let $B=\left[a_{0}\right] \cup A \cup C \cup X$. Since $\left[a_{0}\right]$ intersects $1+p^{n}$ lines of $P G\left(2, p^{n}\right), A$ intersects $\left(p^{n}-p^{d}\right) p^{n}$ new lines, $C$ intersects $\left(p^{n}-p^{d}\right) p^{d}$ new lines, and $X$ intersects $p^{2 d}$ new lines, it follows that $B$ intersects all the lines of $P G\left(2, p^{n}\right)$. It is easily seen that $B$ contains no line of $P G\left(2, p^{n}\right)$ and that $|B|=2\left(p^{n}-p^{d}\right)+p^{d}+1$ $=2 p^{n}-p^{d}+1$. This completes the proof.

The following special case, communicated to the author by L. S. Shapley, shows that Theorem 6 does not provide a minimum.

THEOREM 7. If $n=2 d$, then $P G\left(2, p^{2 d}\right)$ contains a blocking coalition with $1+p^{d}+p^{2 d}$ members.

Proof. The points of any $P G\left(2, p^{d}\right)$ imbedded in $P G\left(2, p^{2 d}\right)$ form such a coalition. For there are $1+p^{d}+p^{2 d}$ lines which are extensions of the lines of the subgeometry, and $p^{2 d}-p^{d}$ additional lines through each point of the subgeometry, making a total of

$$
1+p^{d}+p^{2 d}+\left(1+p^{d}+p^{2 d}\right)\left(p^{2 d}-p^{d}\right)=1+p^{2 d}+p^{4 d} .
$$

Since this accounts for all the lines of $P G\left(2, p^{2 d}\right)$, the coalition blocks.

If $p^{2 d}>4$, this number $1+p^{d}+p^{2 d}$ is less than the number $2 p^{2 d}-p^{d}+1$ provided by Theorem 6 .

The problem of determining the number of points in a mininum blocking coalition remains open. In nongame-theoretic terms, the problem is to find the smallest number of points in a set which intersects every line but contains no entire line. Similar questions can be asked, of course, in the higher dimensional cases, in the nonDesarguesian geometries, and in those block designs in which every pair of distinguished sets intersect.

\section{BIBLIOGRAPHY}

1. R. D. Carmichael, Introduction to the theory of groups of finite order, Ginn, 1937.

2. D. B. Gillies, Some theorems on $n$-person games, Princeton University Doctoral Thesis, 1953, unpublished.

3. R. D. Luce, $A$ definition of stability for $n$-person games, Ann. of Math. vol. 59 (1954) pp. 357-366.

4. J. von Neumann and O. Morgenstern, Theory of games and economic behavior, 2d ed., Princeton, 1947.

5. L. S. Shapley, Lectures on $n$-person games, Princeton University Notes, to be published.

6. J. Singer, $A$ theorem in finite projective geometry and some applications to number theory, Trans. Amer. Math. Soc. vol. 43 (1938) pp. 377-385. 
7. E. Snapper, Periodic transformations of affine and projective geometries, Canadian Journal of Mathematics vol. 2 (1950) pp. 149-151.

8. O. Veblen and W. H. Bussey, Finite projective geometries, Trans. Amer. Math. Soc. vol. 7 (1906) pp. 241-259.

Brooklyn College

\section{NOTE ON LINEAR FORMS}

\section{J. B. ROBERTS}

1. There has been some interest in solutions to the equation

$$
n=a_{0} x_{0}+a_{1} x_{1}+\cdots+a_{s} x_{8}
$$

where the $a_{i}$ are fixed positive integers with $\operatorname{gcd}=1$ and the $x_{i}$ are non-negative integers. In particular the question of finding the smallest $n$ for which all greater integers have a solution has been investigated to some extent $[1 ; 2]$. It seems that the solution for $s=1$ has been known for some time but that the problem in general remains unsolved for $s>1$. In the paper of $\mathrm{A}$. Brauer cited in the bibliography various upper bounds for the smallest $n$ are given and the actual value of the smallest $n$ is determined for the $a_{i}$ consecutive integers. The main result of this paper is the determination of this smallest $n$ when the $a_{i}$ are in arithmetical progression.

2. Our investigation then is with the linear form

$$
F=a_{0} x_{0}+\cdots+a_{8} x_{8} \text {. }
$$

Throughout this paragraph we assume $2 \leqq a_{0}, \operatorname{gcd} a_{i}=1$ and $a_{j}=a_{0}+j d$. Thus the $a_{i}$ are in arithmetical progression. Then we have the

THEOREM. F represents all $n \geqq N$ where

$$
N=\left(\left[\frac{a_{0}-2}{s}\right]+1\right) \cdot a_{0}+(d-1)\left(a_{0}-1\right)
$$

with non-negative $x_{i}$ and does not so represent $N-1$.

The proof of this result breaks down into a series of five lemmas.

LEMMA 1. The only integers represented by $F$ when $x_{0}+\cdots+x_{s}=m$ are $m a_{0}, m a_{0}+d, m a_{0}+2 d, \cdots, m a_{0}+m s d$.

Proof. $F$ represents $m a_{0}$ for $x_{0}=m$, other $x_{i}=0$. If $F$ represents $m a_{0}+k d$ with $\sum_{0}^{s} x_{i}=m$ and $k<m s$ then $x_{i}>0$ for some $i<s$. In the representation of $m a_{0}+k d$ replace $x_{0}, \cdots, x_{i}, x_{i+1}, \cdots, x_{s}$ by $x_{0}, \cdots, x_{i}-1, x_{i+1}+1, \cdots, x_{s}$. Now $F$ represents $m a_{0}+(k+1) d$.

Received by the editors June $28,1955$. 